\title{
Quantification of All-Trans Retinoic Acid by Liquid Chromatography-Tandem Mass Spectrometry and Association with Lipid Profile in Patients with Type 2 Diabetes
}

\author{
Jakob Morgenstern ${ }^{1, *(\mathbb{D})}$, Thomas Fleming ${ }^{1,2}$, Elisabeth Kliemank ${ }^{1}$, Maik Brune ${ }^{1}$, Peter Nawroth ${ }^{1}$ \\ and Andreas Fischer $1,3,4$ (D) \\ 1 Department of Internal Medicine I and Clinical Chemistry, University Hospital Heidelberg, \\ Im Neuenheimer Feld 410, 69120 Heidelberg, Germany; thomas.fleming@med.uni-heidelberg.de (T.F.); \\ elisabeth.kliemank@med.uni-heidelberg.de (E.K.); maik.brune@med.uni-heidelberg.de (M.B.); \\ Peter.nawroth@med.uni-heidelberg.de (P.N.); a.fischer@dkfz.de (A.F.) \\ 2 German Center for Diabetes Research (DZD), 85764 Neuherberg, Germany \\ 3 Division Vascular Signaling and Cancer (A270), German Cancer Research Center (DKFZ), \\ 69120 Heidelberg, Germany \\ 4 European Center for Angioscience, Medical Faculty Mannheim, Heidelberg University, \\ 68167 Mannheim, Germany \\ * Correspondence: jakob.morgenstern@med.uni-heidelberg.de; Fax: +49-6221-565-226
}

Citation: Morgenstern, J.; Fleming,

T.; Kliemank, E.; Brune, M.; Nawroth,

P.; Fischer, A. Quantification of

All-Trans Retinoic Acid by Liquid Chromatography-Tandem Mass Spectrometry and Association with Lipid Profile in Patients with Type 2 Diabetes. Metabolites 2021, 11, 60. https://doi.org/10.3390/ metabo11010060

Received: 16 December 2020

Accepted: 15 January 2021

Published: 19 January 2021

Publisher's Note: MDPI stays neutral with regard to jurisdictional claims in published maps and institutional affiliations.

Copyright: (c) 2021 by the authors. Licensee MDPI, Basel, Switzerland. This article is an open access article distributed under the terms and conditions of the Creative Commons Attribution (CC BY) license (https:// creativecommons.org/licenses/by/ $4.0 /)$.

\begin{abstract}
Retinoic acids are vitamin A metabolites that have numerous essential functions in humans, and are also used as drugs to treat acne and acute promyelocytic leukemia. All-trans retinoic acid (atRA) is the major occurring metabolite of retinoic acid in humans. This study provides a sensitive and specific liquid chromatography-tandem mass spectrometry approach in order to quantify atRA in human plasma samples. The isolation of atRA by hyperacidified liquid-liquid extraction using hexane and ethyl acetate resulted in a recovery of $89.7 \pm 9.2 \%$. The lower limit of detection was $20 \mathrm{pg} \cdot \mathrm{mL}^{-1}$, and 7 point calibration displayed good linearity $\left(\mathrm{R}^{2}=0.994\right)$ in the range of 50-3200 $\mathrm{pg} \mathrm{mL}^{-1}$. Selectivity was guaranteed by the use of two individual mass transitions (qualifier and quantifier), and precision and accuracy were determined intraday and interday with a coefficient variation of $9.3 \%$ (intraday) and $14.0 \%$ (interday). Moreover, the method could be used to isolate atRA from hyperlipidemic samples. Applying this method to plasma samples from patients with poorly controlled Type 2 diabetes significantly decreased atRA plasma levels as compared to those of the healthy controls. In addition, at RA concentrations were highly associated with increased low-density lipoprotein (LDL) and decreased high-density lipoprotein (HDL) cholesterol levels.
\end{abstract}

Keywords: retinoic acid; liquid chromatography-tandem mass spectrometry; hyperlipidemia; liquidliquid extraction

\section{Introduction}

Vitamin A is absorbed in the small intestine as retinol, and its metabolites have various physiological functions during development and for the maintenance of cellular homeostasis. Retinol can be oxidized to retinaldehyde, which is essential for vision, and further to retinoic acids, which play key roles in the regulation of the immune system, cell growth, and cell differentiation. Although several different retinoic acids are synthesized in cells, all-trans retinoic acid (atRA) is the major active metabolite of retinol, which mediates the majority of physiological functions [1]. AtRA is a lipophilic substance that acts through binding to DNA-bound nuclear receptors. This allows for the regulation of over 500 genes depending on cell type [2]. Physiological atRA levels are maintained by regulated synthesis through aldehyde dehydrogenases and degradation by enzymes of the cytochrome P450 family [3]. 
In clinical practice, atRA is used for the treatment of acne (topical application) and acute promyelocytic leukemia (systemically). In blood, atRA undergoes a rapid turnover, and only degradation products, such as 4-oxo-atRA, can be detected in urine $[4,5]$. It is likely that the metabolism of retinoids is affected or may display a causal role in the etiology of various diseases [1,3]. For example, the production rate of atRA is regulated by food intake and insulin [6], while atRA inhibits progression of diabetic nephropathy in a diabetes mouse model by preventing nuclear translocation of NF- $\mathrm{kB}$ in glomeruli and proximal tubules [7]. This indicates the potential role of atRA in the pathogenesis of diabetes mellitus and diabetic complications, which warrants further mechanistic investigations and association studies to determine whether the circulatory levels of atRA levels would be useful as biomarkers predicting diabetic complications.

Several studies over the last 30 years addressed atRA plasma levels in humans using different technical approaches [8-12]. However, such approaches were used for therapeutic drug monitoring in acute promyelocytic leukemia or for determining atRA plasma concentrations in healthy volunteers with less specific and sensitive techniques than what is currently possible $[8,10,13-16]$.

This study aimed at establishing a highly sensitive state-of-the art method in order to quantify plasma concentrations of atRA and to apply it to the analysis of patients with Type 2 diabetes. Here, we report a robust and simple extraction assay for atRA that can be applied to hyperlipidemic plasma samples, a common preanalytical challenge in laboratory medicine, particularly with respect to obese or diabetic patients $[17,18]$. The presented method allows for sufficient recovery, and sensitive and specific quantification. In addition, given atRA instability, this study aimed to assess the conditions under which atRA levels remain constant either in the blood or after extraction.

\section{Results}

\subsection{Fragmentation and Mass Transitions}

The acquisition of atRA was achieved in the positive ionization mode of mass spectrometry. In the negative ionization mode of mass spectrometry, the abundance of precursor and product ion was $\sim 10$-fold lower than that of positive ionization (Supplementary Figure S1). The precursor and product ion scan for atRA revealed fragments of $283.1 \mathrm{~m} / z$ (qualifier) due to water loss, and $122.9 \mathrm{~m} / z$ (quantifier) caused by the loss of the complete side chain of the carbonyl groups (Figure 1A). This was confirmed by the fragmentation patterns of atRA-d5. However, the smaller fragment of $126.8 \mathrm{~m} / z$ (quantifier) displayed a loss in deuterated hydrogen (Figure 1B).

A

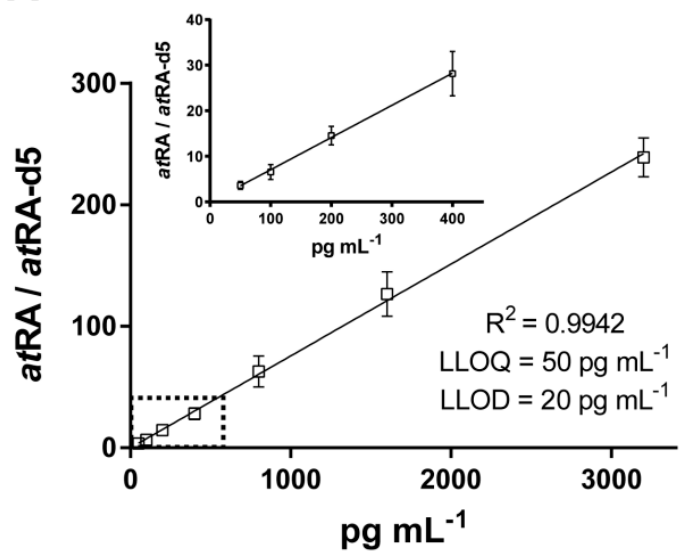

B 100

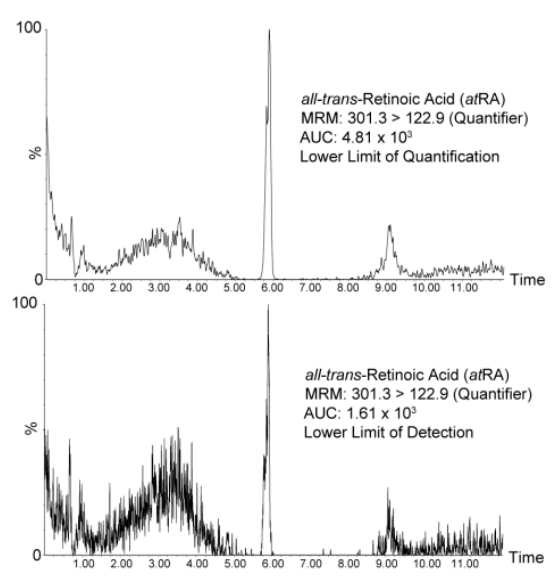

Figure 1. Parameters of linearity and limitations. (A) Calibration range (50-3200 pg. $\left.\mathrm{mL}^{-1}\right)$; lower limit of quantification (LLOQ) (signal-to-noise ratio, 6.4/1); lower limit of detection (LLOD; signal-to-noise ratio, 3.2/1) and coefficient of determination (R2). (B) Total ion chromatogram of all-trans retinoic acid (atRA) for LLOQ (top) and LLOD (bottom). 


\subsection{Chromatography}

The efficient separation of atRA was achieved using a reverse-phase C18 column. The positive ionization of the precursor ions was enhanced by the addition of formic acid. The use of a simple biphasic gradient composed of water and methanol resulted in a chromatogram with a stable peak shape and reproducibility, and a low noise signal (Figure 2C,D). However, to avoid nonspecific peaks (Figure 2C; RT, $\sim 9 \mathrm{~min}$ ) due to a mild carry-over effect, five consecutive blanks composed of water and methanol (1:1) were run every 50 samples.

A

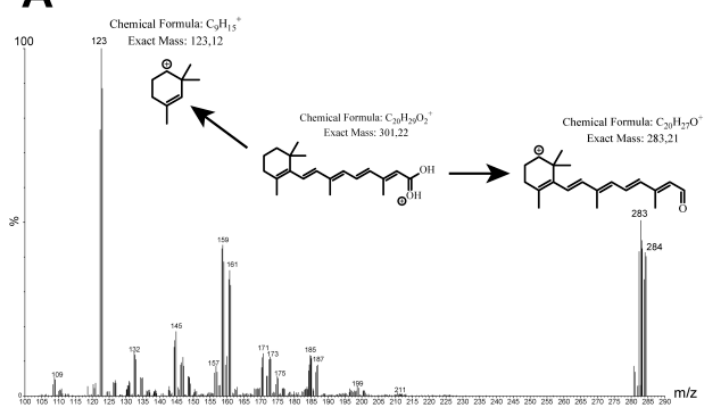

B

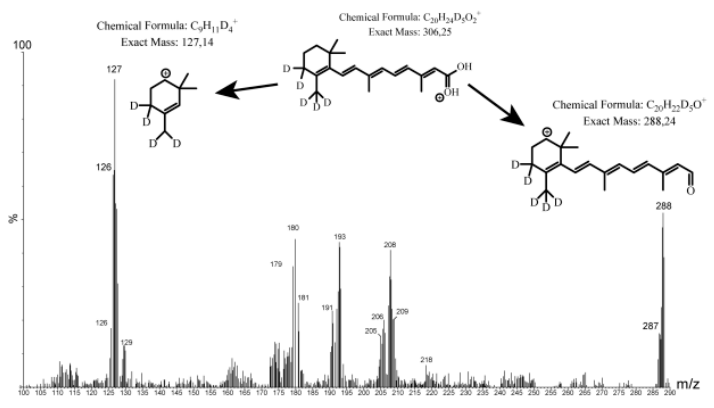

C

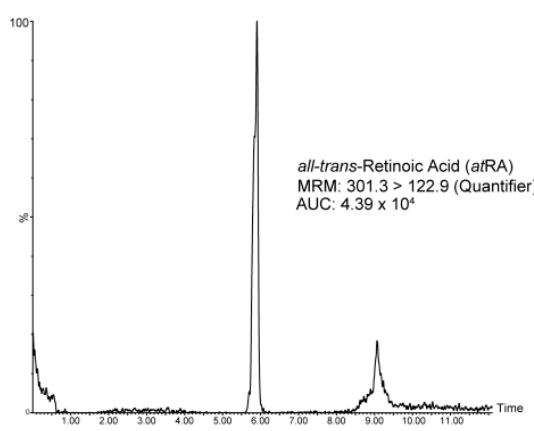

D

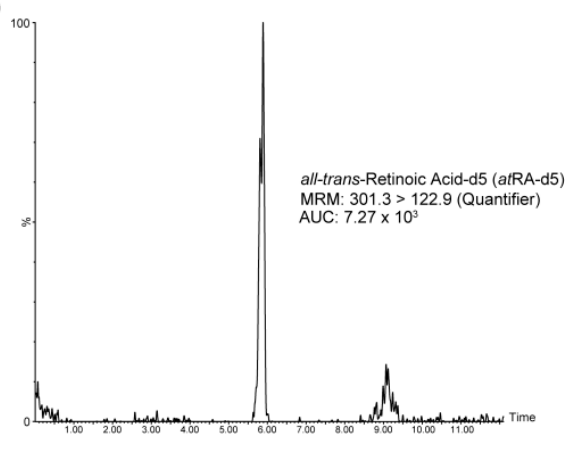

Figure 2. Multiple reaction monitoring (MRM) chromatograms and total ion chromatograms of atRA/atRA-d5. (A) Extracted MRM spectrum (quantifier/qualifier) of atRA. (B) Extracted MRM spectrum (quantifier/qualifier) of atRA-d5. (C) Total ion chromatogram of atRA in a control subject $\left(1.02 \mathrm{ng} \cdot \mathrm{mL}^{-1}\right)$. (D) Total ion chromatogram of atRA-d5 in the same control subject as in (C).

\subsection{Analytical Specificity}

The purity verification of $a t \mathrm{RA}$ and $a t \mathrm{RA}-\mathrm{d} 5$ was provided by the supplier company. The two product ions with the highest intensity were selected as quantifier and qualifier (Table 1). Using artificial human plasma (Biseko ${ }^{\circledR}$, Dreieich, Germany) without endogenously produced atRA, no coeluting compounds were found, which interfered with the detection of either quantifier or qualifier. However, the possibility that any medications could interfere with our assay could not be excluded. Following the optimization of multiple reaction monitoring (MRM), several injections of spiked artificial plasma showed that the fragmentation patterns for qualifier and quantifier of atRA and atRA-d5 were reproducible and stable over a long period of time with acceptable accuracy and precision $(n \geq 100$ injections, Table 2). 
Table 1. Retention time (Rt), mass transition (MRM), cone voltage (COV), and collision energy (CE) for atRA and atRA-d5.

\begin{tabular}{cccccc}
\hline Analytes & $\mathbf{R}_{\mathbf{t}}(\mathbf{m i n})$ & $\begin{array}{c}\text { MRM } \\
\text { Quantifier }(\boldsymbol{m} / \mathbf{z})\end{array}$ & $\begin{array}{c}\text { MRM } \\
\text { Qualifier }(\boldsymbol{m} / \mathbf{z})\end{array}$ & COV (V) & CE (V) \\
\hline atRA & 5.90 & $301.3>122.9$ & $301.1>283.1$ & 23 & 16 \\
atRA-d5 & 5.89 & $306.3>126.8$ & $306.3>288.1$ & 26 & 16 \\
\hline
\end{tabular}

Table 2. Parameters of variability and extraction recovery for atRA. Intra- and interday accuracy/precision and extraction efficiency based on recovery of atRA-d5. Accuracy is the mean of quantified concentration given as percentage of three spiked concentrations (nominal) in human artificial plasma samples. Precision is the coefficient of variation (CV) of mean concentration determined for three different concentration levels (intra/interday, $n=4$ ). The precision of retention time is described as the CV of the mean retention time of 120 injections (21 calibrators, 99 samples) over a period of 65 days. Long-term accuracy and precision were determined over a period of 30 days with 105 injections of varying concentration within the calibration range.

\begin{tabular}{|c|c|c|c|c|c|c|}
\hline Parameter & $\begin{array}{c}\text { Nominal } \\
\text { Concentration } \\
\left(\mathrm{pg} \mathrm{mL} \mathrm{mL}^{-1}\right)\end{array}$ & $\begin{array}{c}\text { Recovery } \\
(\%)\end{array}$ & $\begin{array}{c}\text { Measured } \\
\text { Concentration } \\
\left(\mathrm{pg} \mathrm{mL}^{-1}\right)\end{array}$ & $\begin{array}{c}\text { Accuracy } \\
(\%)\end{array}$ & $\begin{array}{c}\text { Precision } \\
\text { (\% CV) }\end{array}$ & $\begin{array}{c}\text { Precision of } \\
\text { Retention Time } \\
(\% \mathrm{CV})\end{array}$ \\
\hline \multirow{3}{*}{ Intraday } & 50 & $87.4 \pm 8.2$ & $46.8 \pm 4.6$ & 93.6 & 9.8 & \multirow{6}{*}{5.5} \\
\hline & 600 & $90.0 \pm 6.4$ & $618.6 \pm 41.7$ & 103.1 & 6.7 & \\
\hline & 2500 & $91.7 \pm 12.9$ & $2320.4 \pm 263.2$ & 92.8 & 11.3 & \\
\hline \multirow{3}{*}{ Interday } & 50 & & $57.9 \pm 8.3$ & 115.8 & 14.3 & \\
\hline & 600 & & $519.4 \pm 81.2$ & 86.6 & 15.6 & \\
\hline & 2500 & & $2549.6 \pm 307.3$ & 102.0 & 12.1 & \\
\hline Long-term & Various & & & $94.0 \pm 14.2$ & 17.8 & \\
\hline
\end{tabular}

\subsection{Linearity and Determination Limits}

For the 7 point calibration, the ratio between atRA and atRA-d5 was used for quantification. The obtained calibration coefficients $\left(R^{2}\right)$ for 7 point calibration $\left(50-3200 \mathrm{pg} \cdot \mathrm{mL}^{-1}\right)$ were $>0.99$ (Figure 1A). The achieved lower limits of detection and quantification were 20 and $50 \mathrm{pg} \cdot \mathrm{mL}^{-1}$, respectively (Figure $1 \mathrm{~A}, \mathrm{~B}$ ).

\subsection{Recovery, Precision, and Accuracy}

The extraction recovery of atRA-d5 in plasma was $89.7 \pm 9.2 \%$ (Table 2). Methanol precipitation and acidification with hydrochloric acid resulted in the best recovery as compared to other precipitation or acidification reagents such as trichloroacetic acid, ethanol, acetonitrile, or formic acid (data not shown). The precision of the replicate analyses was evaluated for 50,600 , and $2500 \mathrm{pg} \cdot \mathrm{mL}^{-1}$. The coefficient of variation $(\mathrm{CV})$ was intraday at $9.3 \%$ and interday at $14.0 \%$ (Table 2). Accuracy achieved a mean value of $96.5 \%$ on intraday basis and a mean of $101.2 \%$ on interday basis (Table 2). The long-term accuracy and precision of artificial plasma ( $n \geq 100$ samples spiked with varying atRA concentration) were $94.0 \pm 14.2 \%$ and $17.8 \% \mathrm{CV}$, respectively (Table 2 ).

\subsection{Stability}

Following storage in darkness under various conditions, samples were quantified and compared to the quantification directly after extraction. The storage of plasma for 1 week at $4{ }^{\circ} \mathrm{C}$ was associated with a significant reduction in atRA ( 51\%; Table 3). Postprocessing stability in an assay buffer revealed that atRA was degraded when stored for 1 week at $4{ }^{\circ} \mathrm{C}$. Several freeze-thaw cycles were not associated with a significant reduction in atRA ( $14 \%$ reduction; Table 3). Furthermore, exposure to $254 \mathrm{~nm}$ UV light for $3 \mathrm{~h}$ was associated with $\sim 40 \%$ reduction in atRA (Supplementary Table S1). 
Table 3. Pre- and postprocessed sample stability of atRA under varying conditions. Stability is a change in percentage calculated by measured concentrations divided by nominal concentrations (samples were spiked with $1000 \mathrm{pg} \cdot \mathrm{mL}^{-1}$ of atRA; $n=4$ for each test).

\begin{tabular}{ccc}
\hline Conditions & \multicolumn{2}{c}{ Recovery } \\
\cline { 2 - 3 } & Preprocessed & Postprocessed \\
\hline 1 h at $20^{\circ} \mathrm{C}$ & $93 \pm 12 \%$ & \\
$6 \mathrm{~h}$ at $20^{\circ} \mathrm{C}$ & $84 \pm 19 \%$ & \\
$1 \mathrm{~h}$ at $4{ }^{\circ} \mathrm{C}$ & $89 \pm 17 \%$ & $77 \pm 18 \%$ \\
$6 \mathrm{~h}$ at $4{ }^{\circ} \mathrm{C}$ & $81 \pm 21 \%$ & $44 \pm 14 \%$ \\
1 week at $4{ }^{\circ} \mathrm{C}$ & $51 \pm 31 \%$ & $91 \pm 9 \%$ \\
1 week at $-20^{\circ} \mathrm{C}$ & & $93 \pm 16 \%$ \\
1 month at $-20^{\circ} \mathrm{C}$ & $101 \pm 15 \%$ & $82 \pm 22 \%$ \\
\hline F/T stability $(6$ cycles) & & \\
\hline
\end{tabular}

\subsection{Clinical Application}

This method was developed to quantify atRA in human plasma samples. A total of 14 plasma samples had triglyceride levels above $300 \mathrm{mg} \cdot \mathrm{dL}^{-1}$. The best recovery in those samples was achieved following strong acidification with $\mathrm{HCl}(10 \mathrm{M})$, liquid-liquid extraction with a combination of ethyl acetate/hexane (1:1), and the incubation of the sample at $4{ }^{\circ} \mathrm{C}$ for $20 \mathrm{~min}$. No acidification and the usage of different extraction solvents were associated with poor recovery in those hyperlipidemic samples (data not shown). For a constant extraction procedure, this method was applied to all plasma samples.

Using the developed LC-MS/MS method, plasma levels of atRA were found to be in the range of 0.57 to $2.76 \mathrm{ng} \cdot \mathrm{mL}^{-1}$ in all patients. A cohort of patients with poorly controlled Type 2 diabetes displayed hyperlipidemia, increased C-reactive protein, and blood glucose concentration, as well as excessively increased HbA1c (Table 4). In patients with poorly controlled Type 2 diabetes, atRA levels were significantly lower, with $1.38 \pm 0.42 \mathrm{ng} \cdot \mathrm{mL}^{-1}$, as compared to healthy individuals with $1.77 \pm 0.45 \mathrm{ng} \cdot \mathrm{mL}^{-1}(p<0.01$; Figure 3A). In addition, plasma atRA levels were strongly associated with the lipid profiles of the whole cohort $(n=44)$, especially for high-density lipoprotein (HDL; $r=0.598 ; p<0.001)$ and low-density lipoprotein (LDL) cholesterol ( $\mathrm{r}=-0.469 ; p<0.01)$, and also for body-mass index (BMI) and triglyceride concentration (Figure 3B-F). In a cohort of patients with well-adjusted Type 2 diabetes, atRA levels decreased $\left(1.47 \pm 0.37 \mathrm{ng} \cdot \mathrm{mL}^{-1}\right)$, but this was statistically not significant ( $p=0.053$ vs. controls; Supplementary Table S2).

Table 4. Mean baseline characteristics of control and diabetic patient cohorts. All parameters were determined prior to collection. Data are mean $\pm \mathrm{SD}{ }^{* * *} p<0.001$, vs. controls. All other characteristics were not significant $(p>0.05)$.

\begin{tabular}{|c|c|c|}
\hline Parameter- & Controls $(n=20)$ & Type 2 Diabetes $(n=24)$ \\
\hline Sex (\% male) & 63.8 & 65.3 \\
\hline Age (years) & $42.1 \pm 12.3$ & $49.6 \pm 15.5$ \\
\hline Body-mass index (BMI; $\left.\mathrm{kg} \cdot \mathrm{m}^{-2}\right)$ & $24 \pm 2.5$ & $31.3 \pm 6.1^{* * *}$ \\
\hline Blood glucose $\left(\mathrm{mg} \cdot \mathrm{dL}^{-1}\right)$ & $97.1 \pm 12.3$ & $186.3 \pm 84.3^{* * *}$ \\
\hline $\mathrm{HbA} 1 \mathrm{c}(\%)$ & $5.7 \pm 0.8$ & $11.2 \pm 1.6^{* * *}$ \\
\hline $\begin{array}{l}\text { C-reactive protein } \\
\left(\mathrm{CRP} ; \mathrm{mg} \cdot \mathrm{L}^{-1}\right)\end{array}$ & $1.2 \pm 1.1$ & $12.9 \pm 8.6^{* * *}$ \\
\hline Total cholesterol $\left(\mathrm{mg} \mathrm{dL}^{-1}\right)$ & $168.4 \pm 19.9$ & $256.1 \pm 74.5^{* * *}$ \\
\hline Triglycerides $\left(\mathrm{mg} \mathrm{dL}^{-1}\right)$ & $137.7 \pm 24.2$ & $285.3 \pm 86.4^{* * *}$ \\
\hline Low-density lipoprotein (LDL; $\mathrm{mg} \cdot \mathrm{dL}^{-1}$ ) & $82.7 \pm 25.7$ & $167.5 \pm 73.4^{* * *}$ \\
\hline High-density lipoprotein $\left(\mathrm{HDL} ; \mathrm{mg} \cdot \mathrm{dL}^{-1}\right.$ ) & $58.2 \pm 15.9$ & $31.5 \pm 9.1^{* * *}$ \\
\hline
\end{tabular}


A

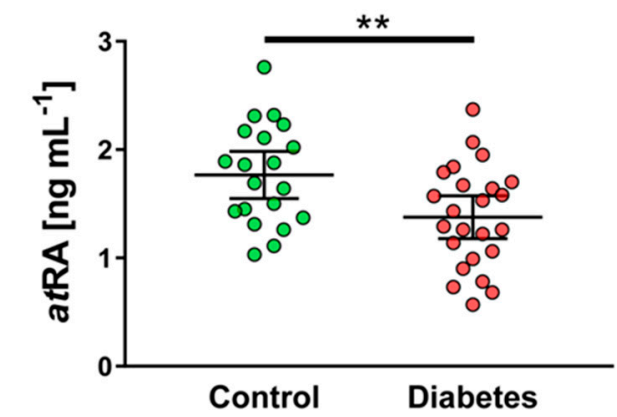

C

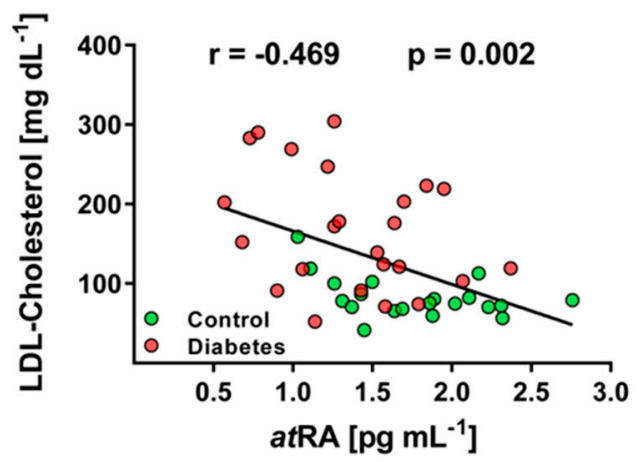

$\mathbf{E}$

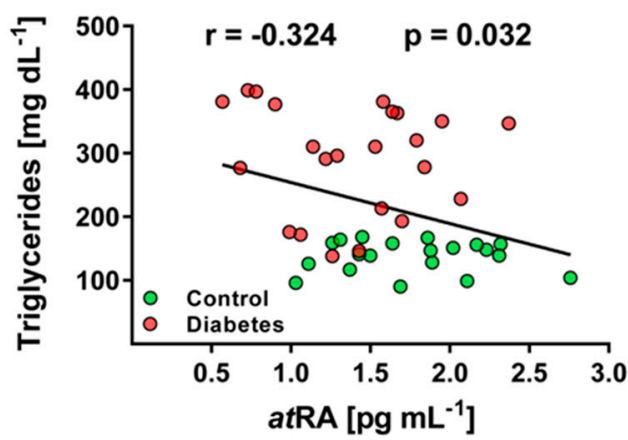

B

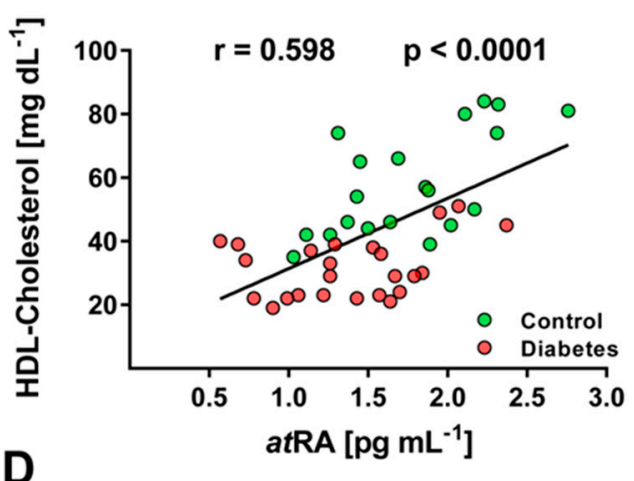

D

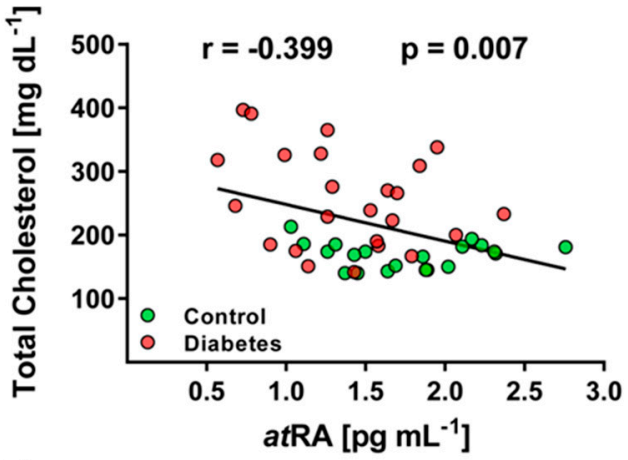

$\mathbf{F}$

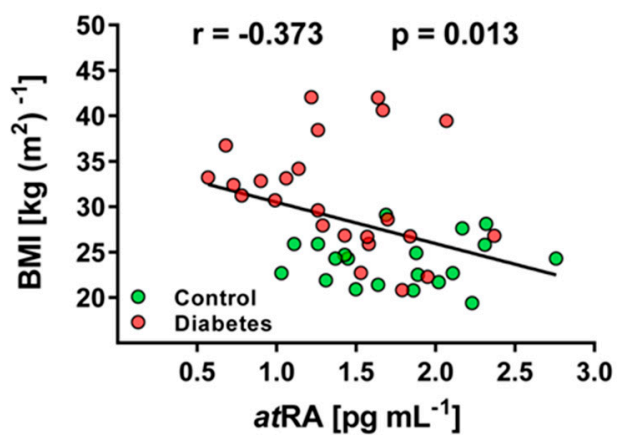

Figure 3. Clinical application and associations with lipid profile in human plasma samples. (A) Quantification of atRA in control patients $(n=20)$ and patients with Type 2 diabetes $(n=24)$. Correlation analysis of $(\mathbf{B})$ atRA and HDL cholesterol, (C) atRA and LDL cholesterol, (D) atRA and total cholesterol, (E) atRA and triglycerides, and (F) atRA and BMI. (B-F) Correlation analysis was performed in the whole cohort. Level of significance and Pearson's correlation coefficient (r) are shown. Data are mean \pm SEM. ${ }^{* *} p<0.01$.

\section{Discussion}

This study offers a sensitive and specific liquid chromatography, tandem mass spectrometry (LC-MS/MS) approach for the quantification of atRA in hyperlipidemic human plasma samples. Within this context, this method for the first time describes the usage of $122.9 \mathrm{~m} / \mathrm{z}$ for the quantification of atRA in biological matrices, which may be a result of a more efficient fragmentation in the collision cell as compared to that of other studies [6,8,19]. As previously described, the separation and ionization of atRA were achieved using a reverse-phase C18 column and formic acid $[8,9,14,20]$. With 20 and $50 \mathrm{pg} \cdot \mathrm{mL}^{-1}$ as the lower limits of detection and quantification, this method is more sensitive than other published methods in the field that use LC-MS and high-performance liquid chromatography (HPLC) approaches [9-11,15,16]. Ex vivo, atRA is chemically unstable, and readily oxidized and 
isomerized when exposed to heat, light, or oxygen $[15,16]$, which reflects the necessity for stability analysis. Pre- and postprocessing analyses revealed that atRA had sufficient short-term stability, but degraded when stored for 1 week at $4{ }^{\circ} \mathrm{C}$, which is in line with previous studies on atRA stability [21]. Exposure to $254 \mathrm{~nm}$ UV light for $3 \mathrm{~h}$ or longer was associated with a significant reduction in atRA. Therefore, the usage of amber reaction tubes and the avoidance of direct sunlight during analysis are strongly recommended.

As a lipophilic compound, atRA presents challenges during extraction, particularly in patients with an altered lipid profile displayed by increased triglycerides, LDL, and total cholesterol. With plasma levels of at RA between 0.57 and $2.76 \mathrm{ng} \cdot \mathrm{mL}^{-1}$ in all patients, our results were in line with those of other studies in healthy individuals, where $0.3-5 \mathrm{ng} \cdot \mathrm{mL}^{-1}$ of atRA was found to be present in the plasma and serum $[8-10,15,16]$. In patients with poorly controlled Type 2 diabetes, lower atRA levels than those of healthy individuals were found. However, a cohort of patients with well-adjusted Type 2 diabetes displayed only a minor decrease in atRA. Future studies should address whether atRA could be causally involved in the progression of diabetes mellitus.

In all analyzed individuals, the plasma atRA levels were strongly associated with lipid profiles and body-mass index (BMI). This confirms a recent study in which atRA was claimed to be a predictive marker for the development of metabolic syndrome, which is strongly associated with the development of Type 2 diabetes and cardiovascular disease [22]. Experimental diabetic model systems showed that atRA can block adipocyte differentiation [23]. Furthermore, atRA affects insulin secretion, which is not surprising given the ubiquitous expression of the retinoic $X$ receptor [24]. Whether the reduction in atRA plasma concentration in dyslipidemic patients is the cause or effect of lipid dysregulation remains unclear and should be addressed in further studies.

\section{Materials and Methods}

\subsection{Chemicals and Reagents}

Acetonitrile, hexane, methanol, hydrochloric acid (37\%), and water were of high purity grade and purchased from Sigma-Aldrich (Steinheim, Germany). Formic acid was purchased from Biosolve (Valkenswaard, Netherlands). All-trans retinoic acid (atRA) and all-trans retinoic acid-d5 (atRA-d5) were purchased as the LC-MS standard (purity $>98 \%$ ) from Biomol (Hamburg, Germany). Amber reaction vials (1.5 mL) were purchased from Chromsystems (Gräfelfing, Germany). Artificial plasma with a standardized protein composition of human plasma (Biseko ${ }^{\circledR}$, Dreieich, Germany) was purchased from Biotest (Dreieich, Germany) and used as a matrix for method development and validation. Biseko ${ }^{\circledR}$ is a virus-inactivated human-plasma substitute that contains the entire spectrum of serum proteins in a standardized active form [25]. It is prepared from plasma pools of at least 1000 individual donations. One liter of Biseko ${ }^{\circledR}$ solution contains $50 \mathrm{~g}$ of total protein, including albumin (31 g), IgG (7.1 g), IgA (1.55 g), IgM (0.48 g), sodium ions (3.56 g), potassium ions $(0.16 \mathrm{~g})$, calcium ions $(0.08 \mathrm{~g})$, magnesium ions $(0.02 \mathrm{~g})$, and chloride ions (3.65 g).

\subsection{Preparation of Calibration Standards}

Stock solutions of atRA and atRA-d5 were prepared in dimethyl sulfoxide at concentrations of 500 (atRA) or $100 \mu \mathrm{g} \cdot \mathrm{mL}^{-1}$ (atRA-d5) and stored at $-80{ }^{\circ} \mathrm{C}$. Working solutions of atRA were prepared in methanol in the range of $500-100 \mu \mathrm{g} \cdot \mathrm{mL}^{-1}$ and stored at $-20^{\circ} \mathrm{C}$. Calibration standards of atRA were $50,100,200,400,800,1600$, and $3200 \mathrm{pg} \cdot \mathrm{mL}^{-1}$ with the addition of $500 \mathrm{pg} \cdot \mathrm{mL}^{-1}$ of atRA-d5 for each calibrator. All stock and working solutions were kept in amber reaction vials to avoid any degradation by ultraviolet light.

\subsection{Sample Collection and Extraction}

Ethylenediaminetetraacetic acid (EDTA) plasma samples were obtained from 20 healthy controls and 39 patients with Type 2 diabetes. All participants were in a fasting state, and patients with Type 2 diabetes were enrolled if diagnosis had been established according to the guidelines of the German Diabetes Association [26]. The study was ap- 
proved by the ethics committee of Heidelberg University Hospital (Project Identification Code S-383/2016). All patient material and data were acquired with formal written informed consent and in agreement with the guidelines of the ethics committee as previously described [19]. The plasma supernatant was directly aliquoted following centrifugation $\left(5 \mathrm{~min}\right.$ at $5000 \times g ; 4{ }^{\circ} \mathrm{C}$ ) of the whole blood and frozen at $-80^{\circ} \mathrm{C}$. Samples were thawed once, and liquid-liquid extraction was carried out under reduced light condition in amber tubes. The internal standard (100 pg of atRA-d5) was added to $200 \mu \mathrm{L}$ of plasma, which was acidified with $5 \mu \mathrm{L}$ of hydrochloric acid $(10 \mathrm{M})$. Afterwards, $400 \mu \mathrm{L}$ of methanol was added for protein precipitation. After centrifugation $\left(10 \mathrm{~min}\right.$ at $\left.10,000 \times g ; 4{ }^{\circ} \mathrm{C}\right)$, the supernatant was transferred into a new amber tube and $300 \mu \mathrm{L}$ of hexane and $300 \mu \mathrm{L}$ of ethyl acetate was added, which was then mixed for $10 \mathrm{~s}$. The mixture was left for $20 \mathrm{~min}$ at $4{ }^{\circ} \mathrm{C}$ in darkness, and the aqueous and organic phase was separated by centrifugation (10 min at $\left.10,000 \times g ; 4^{\circ} \mathrm{C}\right)$. The upper organic phase was evaporated using a vacuum concentrator (Eppendorf Concentrator Plus) at room temperature. The residue was resuspended in $60 \mu \mathrm{L}$ of methanol/water (2:1) and transferred into an HPLC vial (Waters, Eschborn, Germany).

\subsection{Chromatography}

All analyses were performed on a Waters ${ }^{\circledR}$ Acquity UPLC class I system (Waters, Eschborn, Germany) equipped with a binary solvent-delivery system with an online degasser and a column manager containing a column oven connected to a UPLC autosampler. AtRA was separated by reverse-phase LC on a Waters ${ }^{\circledR}$ Acquity BEH C18 column $(1.7 \mu \mathrm{M}$, $2.1 \times 50 \mathrm{~mm}$ ) at a flow rate of $0.2 \mathrm{~mL} \cdot \mathrm{min}^{-1}$ and a column temperature of $20^{\circ} \mathrm{C}$. During analyses, all samples were stored in the autosampler at a temperature of $4{ }^{\circ} \mathrm{C}$, and the injection volume for each sample varied between 10 and $30 \mu \mathrm{L}$. Solvent A consisted of $0.1 \%$ formic acid in water, and Solvent B was $0.1 \%$ formic acid in methanol. For each run, gradient elution was performed. Solvent A was decreased from $80 \%$ to $0 \%$ (0-4 min), remained isocratic at $0 \%(4-6 \mathrm{~min})$, and increased back to $80 \%$ (6-15 $\mathrm{min})$. The column eluent was directed into the MS, and analyses were performed using MassLynx XS software.

\subsection{Mass Spectrometry}

AtRA detection was performed on a XEVO TQ-S tandem quadrupole mass spectrometer (Waters ${ }^{\circledR}$ ) equipped with an electrospray ionization source (ESI) operated in positive ion mode. Analyte detection was performed using multiple reaction monitoring (MRM). Source parameters were set as follows: capillary voltage, $3.5 \mathrm{kV}$; desolvation temperature, $350{ }^{\circ} \mathrm{C}$; desolvation gas flow, $850 \mathrm{~L} \cdot \mathrm{h}^{-1}$; source temperature, $200{ }^{\circ} \mathrm{C}$; cone gas flow, $250 \mathrm{~L} \cdot \mathrm{h}^{-1}$; collision gas flow, $0.15 \mathrm{~mL} \cdot \mathrm{min}^{-1}$; nebulizer gas flow, 5 bar. Cone and collision voltage were individually optimized for atRA and atRA-d5 and are summarized in Table 1. Acquisition and quantification were completed with MassLynx 4.1 and TargetLynx 2.7.

\subsection{Validation Procedure}

The method was validated for selectivity, matrix effects, linearity, lower limits of detection (LLOD) and quantification (LLOQ), recovery, stability, precision, and accuracy (intra/interday). Seven-point calibration was performed using linear regression. For all concentration calculations, the area ratio of atRA/atRA-d5 was plotted against nominal calibrator concentration. Blank samples (without analytes) were measured for each calibration curve to ensure reliability. For the determination of recovery, LLOD, LLOQ, precision, and accuracy, $200 \mu \mathrm{L}$ of spiked artificial plasma was used. Recovery, precision, and accuracy were validated in an intraday assay using three different concentrations (50, 600, $\left.2500 \mathrm{pg} \cdot \mathrm{mL}^{-1}\right)$, which were measured in quadruplicates. For interday variability, the same concentrations $\left(50,600,2500 \mathrm{pg} \cdot \mathrm{mL}^{-1}\right)$ of spiked artificial plasma were quantified on 3 consecutive days in quadruplicates. LLOD and LLOQ were determined with a signalto-noise ratio of 6.4 (LLOQ) and 3.2 (LLOD). Stability was validated in human artificial plasma (preprocessed) and in assay buffer (postprocessed) at various temperature levels and for different durations by spiking $200 \mu \mathrm{L}$ of plasma sample with 1000 pg of atRA. 
AtRA stability was also assessed for exposure to UV light (254 nm) using transparent tubes. Matrix effects were defined as a suppression of or increase in signal intensity for the chosen MRMs (matrix effects while ionization) or as an increase or decrease in recovery of atRA-d5 (matrix effect while extraction).

\subsection{Clinical Chemistry}

Blood was drawn under fasting conditions as described above, and samples were immediately processed in the accredited Central Laboratory of Heidelberg University Hospital. Plasma levels of total cholesterol, HDL cholesterol, triglycerides, blood glucose, and C-reactive protein were analyzed with clinical chemistry automation (AdviaXPT ${ }^{\circledR} 2400$ chemistry analyzer, Siemens Healthineers, Erlangen, Germany) according to the appropriate standard operating protocol. $\mathrm{HbA1c}$ quantification was performed on whole-blood EDTA samples, and analyses were executed using a VARIANT II HbA1c analyzer (BioRad, Hercules, CA, USA) that utilizes cation-exchange high-performance liquid chromatography. LDL cholesterol was determined using the Friedewald formula as previously described [27].

\subsection{Statistical Analysis}

Statistical-data analysis was performed using GraphPad Prism 7 (GraphPad Software Inc., San Diego, CA, USA). All data are expressed as mean values \pm standard error (SE), and were analyzed for significance using an unpaired $t$ test with Welch's correction. Linear regression and Pearson's correlation coefficient were used to study the association of atRA and BMI, total cholesterol, LDL cholesterol, HDL cholesterol, and triglycerides.

\section{Conclusions}

In this study, atRA was quantified in human-plasma samples using a robust and sensitive LC-MS/MS method that achieved convincing analytical parameters of accuracy, precision, recovery, and stability. Within this context, atRA was isolated from hyperlipidemic human-plasma samples, and results showed a decrease in atRA levels in poorly controlled patients with Type 2 diabetes, and a strong correlation with LDL and HDL cholesterol. In summary, this study adds valuable content for further analytical determinations of atRA using LC-MS/MS, and indicates altered metabolism of retinoids in patients with diabetes mellitus.

Supplementary Materials: The following are available online at https:/ /www.mdpi.com/2218-198 9/11/1/60/s1. Figure S1: Comparison of ionization mode of mass spectrometry for atRA (100 pmol direct injection on column), Table S1: Assessment of atRA stability upon UV-exposure, Table S2: Mean baseline characteristics and atRA levels of the control group (as shown in Table 4) and patients with well-adjusted / poorly controlled type 2 diabetes.

Author Contributions: Experiment design, J.M., E.K., T.F., and A.F.; data collection and analysis, J.M., T.F., A.F., and M.B.; drafting manuscript, J.M., P.N., and A.F. All authors have read and agreed to the published version of the manuscript.

Funding: This research was funded by the Deutsche Forschungsgemeinschaft, project number 394046768 for the SFB1366 project C4 to A.F., and SFB 1118 and GRK 1874 to T.F. and P.N.).

Institutional Review Board Statement: The study was conducted according to the guidelines of the Declaration of Helsinki, and approved by Heidelberg University Hospital (S-383/2016, September 2016).

Informed Consent Statement: Informed consent was obtained from all subjects involved in the study.

Data Availability Statement: The data presented in this study are available on request from the corresponding author. The data are not publicly available due to privacy and/or ethical concerns.

Conflicts of Interest: The authors declare no conflict of interest. The funders had no role in the design of the study; in the collection, analyses, or interpretation of data; in the writing of the manuscript, or in the decision to publish the results. 


\section{References}

1. Marill, J.; Idres, N.; Capron, C.; Nguyen, E.; Chabot, G. Retinoic Acid Metabolism and Mechanism of Action: A Review. Curr. Drug Metab. 2003, 4, 1-10. [CrossRef] [PubMed]

2. Cifelli, C.J.; Ross, A.C. All-trans-retinoic acid distribution and metabolism in vitamin A-marginal rats. Am. J. Physiol. Gastrointest. Liver Physiol. 2006, 291, G195-G202. [CrossRef] [PubMed]

3. Kedishvili, N.Y. Enzymology of retinoic acid biosynthesis and degradation: Thematic Review Series: Fat-Soluble Vitamins: Vitamin A. J. Lipid. Res. 2013, 54, 1744-1760. [CrossRef] [PubMed]

4. Smith, M.A.; Parkinson, D.R.; Cheson, B.D.; Friedman, M.A. Retinoids in cancer therapy. J. Clin. Oncol. 1992, 10, 839-864. [CrossRef]

5. Goldfarb, M.T.; Ellis, C.N.; Voorhees, J.J. Retinoids in Dermatology. Mayo Clin. Proc. 1987, 62, 1161-1164. [CrossRef]

6. Obrochta, K.M.; Krois, C.R.; Campos, B.; Napoli, J.L. Insulin regulates retinol dehydrogenase expression and all-trans-retinoic acid biosynthesis through FoxO1. J. Biol. Chem. 2015, 290, 7259-7268. [CrossRef]

7. Sierra-Modragon, E.; Molina-Jijon, E.; Namorado-Tonix, C.; Rodriguez-Munoz, R.; Pedraza-Chaverri, J.; Reyes, J.L. All-trans retinoic acid ameliorates inflammatory response mediated by TLR4/NF-kB during initiation of diabetic nephropathy. J. Nutr. Biochem. 2018, 60, 47-60. [CrossRef]

8. Arnold, S.L.M.; Amory, J.K.; Walsh, T.J.; Isoherranen, N. A sensitive and specific method for measurement of multiple retinoids in human serum with UHPLC-MS/MS. J. Lipid. Res. 2012, 53, 587-598. [CrossRef]

9. De Leenheer, A.P.; Lambert, W.E.; Claeys, I. All-trans-retinoic acid: Measurement of reference values in human serum by high performance liquid chromatography. J. Lipid. Res. 1982, 23, 1362-1367. [CrossRef]

10. Gundersen, T.E.; Bastani, N.E.; Blomhoff, R. Quantitative high-throughput determination of endogenous retinoids in human plasma using triple-stage liquid chromatography/tandem mass spectrometry. Rapid. Commun. Mass. Spectrom. 2007, 21, 1176-1186. [CrossRef]

11. Teerlink, T.; Copper, M.P.; Klaassen, I.; Braakhuis, B.J. Simultaneous analysis of retinol, all-trans- and 13-cis-retinoic acid and 13-cis-4-oxoretinoic acid in plasma by liquid chromatography using on-column concentration after single-phase fluid extraction. J. Chromatogr. B Biomed. Sci. Appl. 1997, 694, 83-92. [CrossRef]

12. Liu, Y.; Chen, H.; Wang, J.; Zhou, W.; Sun, R.; Xia, M. Association of serum retinoic acid with hepatic steatosis and liver injury in nonalcoholic fatty liver disease. Am. J. Clin. Nutr. 2015, 102, 130-137. [CrossRef] [PubMed]

13. Muindi, J.R.; Frankel, S.R.; Huselton, C.; DeGrazia, F.; Garland, W.A.; Young, C.W.; Warrell, R.P. Clinical pharmacology of oral all-trans retinoic acid in patients with acute promyelocytic leukemia. Cancer Res. 1992, 52, 2138-2142. [PubMed]

14. Eckhoff, C.; Nau, H. Identification and quantitation of all-trans- and 13-cis-retinoic acid and 13-cis-4-oxoretinoic acid in human plasma. J. Lipid. Res. 1990, 31, 1445-1454. [CrossRef]

15. De Ruyter, M.G.; Lambert, W.E.; De Leenheer, A.P. Retinoic acid: An endogenous compound of human blood. Unequivocal demonstration of endogenous retinoic acid in normal physiological conditions. Anal. Biochem. 1979, 98, 402-409. [CrossRef]

16. Napoli, J.L.; Pramanik, B.C.; Williams, J.B.; Dawson, M.I.; Hobbs, P.D. Quantification of retinoic acid by gas-liquid chromatography-mass spectrometry: Total versus all-trans-retinoic acid in human plasma. J. Lipid. Res. 1985, 26, 387-392. [CrossRef]

17. Nikolac, N. Lipemia: Causes, Interference Mechanisms, Detection and Management. Biochem. Med. 2014, 24, 57-67. [CrossRef]

18. Jialal, I. A Practical Approach to the Laboratory Diagnosis of Dyslipidemia. Am. J. Clin. Pathol. 1996, 106, 128-138. [CrossRef]

19. Morgenstern, J.; Fleming, T.; Kadiyska, I.; Brings, S.; Groener, J.B.; Nawroth, P.; Hecker, M.; Brune, M. Sensitive mass spectrometric assay for determination of 15-deoxy- $\Delta 12,14$-prostaglandin $\mathrm{J} 2$ and its application in human plasma samples of patients with diabetes. Anal. Bioanal. Chem. 2018, 410, 521-528. [CrossRef]

20. Kane, M.A.; Chen, N.; Sparks, S.; Napoli, J.L. Quantification of endogenous retinoic acid in limited biological samples by LC/MS/MS. Biochem. J. 2005, 388, 363-369. [CrossRef]

21. Peng, J.-B.; Luo, C.-H.; Wang, Y.-C.; Huang, W.H.; Chen, Y.; Zhou, H.H.; Tan, Z.R. Validation of a Liquid ChromatographyElectrospray Ionization-Tandem Mass Spectrometry Method for Determination of All-Trans Retinoic Acid in Human Plasma and Its Application to a Bioequivalence Study. Molecules 2014, 19, 1189-1200. [CrossRef] [PubMed]

22. Liu, Y.; Chen, H.; Mu, D.; Fan, J.; Song, J.; Zhong, Y.; Li, D.; Xia, M. Circulating Retinoic Acid Levels and the Development of Metabolic Syndrome. J. Clin. Endocrinol. Metab. 2016, 101, 1686-1692. [CrossRef] [PubMed]

23. Rhee, E.-J.; Plutzky, J. Retinoid Metabolism and Diabetes Mellitus. Diabetes Metab. J. 2012, 36, 167. [CrossRef] [PubMed]

24. Pan, J.; Guleria, R.; Zhu, S.; Baker, K. Molecular Mechanisms of Retinoid Receptors in Diabetes-Induced Cardiac Remodeling. J. Clin. Med. 2014, 3, 566-594. [CrossRef] [PubMed]

25. Maier, M.; Reinert, M.; Lehnert, M.; Bauer, C.; Marzi, I. Perioperative Application of a Serum Protein Solution (Biseko ${ }^{\circledR}$ ) After Proximal Femur Fracture of Elder Patients. Eur. J. Trauma Emerg. S 2007, 33, 395-400. [CrossRef] [PubMed]

26. Nauck, M.; Gerdes, C.; Petersmann, A.; Müller-Wieland, D.; Müller, U.A.; Freckmann, G.; Heinemann, L.; Schleicher, E.; Landgraf, R. Definition, Klassifikation und Diagnostik des Diabetes mellitus: Update 2020. Diabetol. Und Stoffwechs. 2020, 15, S9-S17. [CrossRef]

27. Knopfholz, J.; Disserol, C.C.D.; Pierin, A.J.; Schirr, F.L.; Streisky, L.; Takito, L.L.; Massucheto Ledesma, P.; Faria-Neto, J.R.; Olandoski, M.; da Cunha, C.L.P.; et al. Validation of the Friedewald Formula in Patients with Metabolic Syndrome. Cholesterol 2014, 1-5. [CrossRef] 